\title{
Author Correction: MRI Vessel Wall Imaging and Treatment of an Aneurysm at the Atlanto-Axial Segment of an Aber- rant Vertebral Artery
}

Chun On Anderson Tsang, MD¹, Wan Lung Ryo Yeung, $\mathrm{MD}^{2}$, Ka Fung Mak, MD², Wai Man Lui, MD

${ }^{1}$ Division of Neurosurgery, Department of Surgery, Queen Mary Hospital, The University of Hong Kong, Hong Kong

${ }^{2}$ Li Ka Shing Faculty of Medicine, The University of Hong Kong, Hong Kong

${ }^{3}$ Department of Diagnostic Radiology, Queen Mary Hospital, The University of Hong Kong, Hong Kong

Correction to: Neurointervention https://doi.org/10.5469/neuroint.2018.13.1.62, published on March, 2018, Neurointervention 2018;13:62-65

The originally published version of this Article contained an error in the author list: the last and first names of all authors are inverted.

Before correction: Tsang Chun On Anderson, MD', Yeung Wan Lung Ryo, MD², Mak Ka Fung, MD³, Lui Wai Man, MD

After correction: Chun On Anderson Tsang, MD1, Wan Lung Ryo Yeung, MD², Ka Fung Mak, MD³, Wai Man Lui, MD 